Homology, Homotopy and Applications, vol.11(2), 2009, pp.309-326

\title{
LA CATÉGORIE CUBIQUE AVEC CONNEXIONS EST UNE CATÉGORIE TEST STRICTE
}

\author{
GEORGES MALTSINIOTIS
}

(communicated by Ronald Brown)

\begin{abstract}
The aim of this paper is to prove that the category of cubes with connections, introduced by R. Brown and Ph.J. Higgins, is a strict test category in Grothendieck's sense. In particular this implies that cubical sets with connections are models for homotopy types in a very precise sense, in a way that is compatible with the cartesian product.
\end{abstract}

\section{Introduction}

Dans la «Poursuite des Champs» [12], Grothendieck a introduit la notion de catégorie test. Si $A$ est une catégorie test, la catégorie $\widehat{A}$ des préfaisceaux sur $A$ modélise les types d'homotopie, et on dit que $A$ est une catégorie test stricte si le type d'homotopie modélisé par un produit cartésien de deux préfaisceaux est le produit des types d'homotopie correspondants. Dans loc. cit., Grothendieck affirme, sans preuve, que la catégorie des cubes est une catégorie test stricte. En réalité, la catégorie des cubes est bien une catégorie test [8], mais elle n'est pas une catégorie test stricte. On verra par exemple dans la section 6 que le type d'homotopie du produit de deux segments dans la catégorie des ensembles cubiques est isomorphe à celui de $S^{2} \vee S^{1}$. Ce contre-exemple était connu par D.M. Kan, et était l'une des raisons l'ayant conduit à abandonner la théorie des ensembles cubiques, initiée dans [21], au profit de celle des ensembles simpliciaux. Dans cet article, on montre qu'en élargissant la catégorie des cubes par l'ajout de nouvelles dégénérescences, les « morphismes de connexion » introduits par R. Brown et Ph.J. Higgins [4], on obtient bien une catégorie test stricte. Ainsi, conformément à la philosophie de Grothendieck, la catégorie des ensembles cubiques avec connexions est « aussi bonne pour faire de l'homotopie » que celle des ensembles simpliciaux.

Le fait que la catégorie cubique avec connexions se comporte mieux que la catégorie cubique classique a déjà été observé par plusieurs auteurs. Par exemple, A.P. Tonks a montré que les objets groupes de la catégorie des ensembles cubiques avec connexions sont de Kan (satisfont à la propriété d'extension relativement aux inclusions des cornets cubiques) [24], ce qui n'est pas toujours le cas pour les groupes cubiques sans

Received June 26, 2009, revised October 4, 2009; published on December 1, 2009. 2000 Mathematics Subject Classification: 18G55, 18D10, 18E35, 55P15, 55 U05.

Key words and phrases: catégorie test, connexion, ensemble cubique, homotopie.

This article is available at http ://intlpress.com/HHA/v11/n2/a15

Copyright (C) 2009, International Press. Permission to copy for private use granted. 
connexions. Par ailleurs, R. Brown et Ph.J. Higgins [7] ont prouvé un théorème du type Dold-Kan pour les ensembles cubiques avec connexions, qui à nouveau n'est pas vrai pour les ensembles cubiques classiques. Les résultats du présent article suggèrent une explication de ces différences par la compatibilité ou la non compatibilité du produit cartésien aux types d'homotopie représentés.

Les ensembles cubiques avec connexions jouent un rôle important dans une série de travaux de R. Brown et Ph.J. Higgins, qui commence avec [4], et se poursuit avec [5] et [6], ainsi que dans un article du second de ces auteurs [13]. Les connexions sont également cruciales pour la comparaison de l'approche cubique et de l'approche globulaire des $\infty$-catégories [1]. Les ensembles cubiques avec connexions sont étudiés aussi par R. Antolini dans [2], où elle construit ce que Grothendieck appelle des foncteurs test. Une variante plus symétrique est étudiée en profondeur par M. Grandis et L. Mauri dans [11]. Encore une autre variante a été proposée récemment par S.B Isaacson $[\mathbf{1 7}, \mathbf{1 8}]$.

Dans la deuxième section, on rappelle la définition de la catégorie cubique avec connexions, et on démontre quelques propriétés élémentaires utiles pour la compréhension de la suite, et présentant un intérêt indépendant. La troisième section est consacrée aux rappels sur les catégories test de Grothendieck. Pour une étude approfondie de cette théorie, voir [12], ou [22]. Dans la quatrième, on prouve que la catégorie cubique avec connexions est une catégorie test stricte. Le but de la cinquième section est de faire le lien avec la théorie des segments multiplicatifs de Grothendieck. On montre en particulier que la catégorie des cubes avec connexions est la catégorie monoïdale stricte universelle abritant un « cylindre multiplicatif », variante de la notion de segment multiplicatif. Cela constitue peut-être la vraie raison de ses « bonnes » propriétés homotopiques. Dans la dernière section, on explique pourquoi la catégorie cubique sans connexions n'est pas une catégorie test stricte.

\section{La catégorie cubique avec connexions}

2.1. Pour tout entier $n \geqslant 0$, on note $\square_{n}$ l'ensemble ordonné produit $\{0<1\}^{n}$. Pour $n \geqslant 1,1 \leqslant i \leqslant n$, et $\varepsilon=0,1$, on définit une application

$$
\delta_{n}^{i, \varepsilon}: \square_{n-1} \longrightarrow \square_{n}
$$

par la formule

$$
\delta_{n}^{i, \varepsilon}\left(x_{1}, \ldots, x_{n-1}\right)=\left(x_{1}, \ldots, x_{i-1}, \varepsilon, x_{i}, \ldots, x_{n-1}\right),
$$

et pour $n \geqslant 0,1 \leqslant i \leqslant n+1$, une application

$$
\sigma_{n}^{i}: \square_{n+1} \longrightarrow \square_{n}
$$

par la formule

$$
\sigma_{n}^{i}\left(x_{1}, \ldots, x_{n+1}\right)=\left(x_{1}, \ldots, x_{i-1}, x_{i+1}, \ldots, x_{n+1}\right) .
$$

La catégorie des cubes $\square$ est la sous-catégorie de la catégorie des ensembles ordonnés dont les objets sont les ensembles ordonnés $\square_{n}, n \geqslant 0$, et dont les morphismes sont 
engendrés par les applications croissantes

$$
\delta_{n}^{i, \varepsilon}, \quad n \geqslant 1,1 \leqslant i \leqslant n, \varepsilon=0,1, \quad \text { et } \quad \sigma_{n}^{i}, \quad n \geqslant 0,1 \leqslant i \leqslant n+1 .
$$

On vérifie facilement qu'une application

$$
f: \square_{m} \longrightarrow \square_{n}, \quad f=\left(f_{1}, \ldots, f_{n}\right), \quad f_{j}: \square_{m} \longrightarrow \square_{1}, \quad 1 \leqslant j \leqslant n,
$$

est un morphisme de $\square$ si et seulement si elle satisfait aux deux conditions suivantes :

(a) pour tout $j, 1 \leqslant j \leqslant n, f_{j}$ est une application constante (de valeur 0 ou 1 ), ou bien une projection du type

$$
p r_{i}: \square_{m} \longrightarrow \square_{1}, \quad\left(x_{1}, \ldots, x_{m}\right) \longmapsto x_{i}, \quad 1 \leqslant i \leqslant m ;
$$

(b) pour tous $j_{1}, j_{2}, 1 \leqslant j_{1}<j_{2} \leqslant n$, si $f_{j_{1}}=p r_{i_{1}}$ et $f_{j_{2}}=p r_{i_{2}}$, alors $i_{1}<i_{2}$.

Pour $n \geqslant 1,1 \leqslant i \leqslant n$, on définit une application

$$
\gamma_{n}^{i}: \square_{n+1} \longrightarrow \square_{n}
$$

par la formule

$$
\gamma_{n}^{i}\left(x_{1}, \ldots, x_{n+1}\right)=\left(x_{1}, \ldots, x_{i-1}, \sup \left\{x_{i}, x_{i+1}\right\}, x_{i+2}, \ldots, x_{n+1}\right) .
$$

La catégorie des cubes avec connexions $\square^{\mathrm{c}}$ est la sous-catégorie de la catégorie des ensembles ordonnés dont les objets sont les ensembles ordonnés $\square_{n}, n \geqslant 0$, et dont les morphismes sont engendrés par les applications croissantes

$$
\begin{array}{ll}
\delta_{n}^{i, \varepsilon}, & n \geqslant 1, \quad 1 \leqslant i \leqslant n, \quad \varepsilon=0,1, \\
\sigma_{n}^{i}, & n \geqslant 0, \quad 1 \leqslant i \leqslant n+1, \\
\gamma_{n}^{i}, & n \geqslant 1, \quad 1 \leqslant i \leqslant n .
\end{array}
$$

La catégorie des ensembles cubiques (resp. des ensembles cubiques avec connexions) est la catégorie $\widehat{\boldsymbol{\square}}\left(\right.$ resp. $\left.\widehat{\boldsymbol{\square}}^{\mathrm{c}}\right)$ des préfaisceaux sur $\boldsymbol{\square}$ (resp. sur $\boldsymbol{\square}^{\mathrm{c}}$ ).

2.2. Les catégories $\square$ et $\square^{c}$ n'admettent pas des produits binaires, mais il est facile de vérifier que le produit cartésien d'ensembles ordonnés et d'applications croissantes induit des bifoncteurs

$$
\square \times \square \stackrel{\otimes}{\longrightarrow} \square \quad \text { et } \square^{\mathrm{c}} \times \mathbf{\square}^{\mathrm{c}} \stackrel{\otimes}{\longrightarrow} \mathbf{\square}^{\mathrm{c}},
$$

faisant de $\square$ et $\square^{\mathrm{c}}$ des catégories monö̈dales strictes, d'objet unité $\square_{0}$. De façon explicite, pour tous entiers $m, n \geqslant 0$, on a $\square_{m} \otimes \square_{n}=\square_{m+n}$, et si $f: \square_{m} \longrightarrow \square_{m^{\prime}}$ et $g: \square_{n} \longrightarrow \square_{n^{\prime}}$ sont deux flèches de $\square$ (resp. $\square^{\mathrm{c}}$ ), la flèche $f \otimes g: \square_{m+n} \longrightarrow \square_{m^{\prime}+n^{\prime}}$ de $\square\left(\right.$ resp. $\boldsymbol{\square}^{\mathrm{c}}$ ) est définie par la formule

$$
f \otimes g\left(x_{1}, \ldots, x_{m+n}\right)=\left(f\left(x_{1}, \ldots, x_{m}\right), g\left(x_{m+1}, \ldots, x_{m+n}\right)\right) .
$$

Proposition 2.3. La catégorie $\square^{\mathrm{c}}$ est la catégorie dont les objets sont les ensembles ordonnés $\square_{n}, n \geqslant 0$, et dont les flèches sont les applications

$$
f: \square_{m} \longrightarrow \square_{n}, \quad f=\left(f_{1}, \ldots, f_{n}\right), \quad f_{j}: \square_{m} \longrightarrow \square_{1}, \quad 1 \leqslant j \leqslant n,
$$

satisfaisant aux deux conditions suivantes : 
(a) pour tout $j, 1 \leqslant j \leqslant n, f_{j}$ est une application constante (de valeur 0 ou 1 ), ou bien il existe un sous-ensemble non vide $E$ de $\{1,2, \ldots, m\}$ tel que $f_{j}=\sup _{E}$, autrement dit, $f_{j}$ est définie par la formule

$$
f_{j}\left(x_{1}, \ldots, x_{m}\right)=\sup \left\{x_{i} \mid i \in E\right\} .
$$

(b) pour tous $j_{1}, j_{2}, 1 \leqslant j_{1}<j_{2} \leqslant n$, si $f_{j_{1}}=\sup _{E_{1}}$ et $f_{j_{2}}=\sup _{E_{2}}$, où $E_{1}$ et $E_{2}$ sont deux parties non vides de $\{1,2, \ldots, m\}$, alors $\max \left(E_{1}\right)<\min \left(E_{2}\right)$.

Démonstration. Il est facile de vérifier que les applications satisfaisant aux conditions $(a)$ et $(b)$ sont stables par composition, et que les générateurs $\delta_{n}^{i, \varepsilon}, \sigma_{n}^{i}, \gamma_{n}^{i}$ satisfont à ces conditions, ce qui prouve qu'il en est de même pour toute flèche de $\mathbb{\square}^{\mathrm{c}}$. Réciproquement, soit

$$
f: \square_{m} \longrightarrow \square_{n}, \quad f=\left(f_{1}, \ldots, f_{n}\right), \quad f_{j}: \square_{m} \longrightarrow \square_{1}, \quad 1 \leqslant j \leqslant n,
$$

une application satisfaisant aux conditions $(a)$ et $(b)$. On va montrer par récurrence sur $n$ que $f$ est alors une flèche de $\boldsymbol{\square}^{\mathrm{c}}$. Si $n=0$, comme $\square_{0}$ est un singleton, on a forcément $f=\sigma_{0}^{1} \circ \cdots \circ \sigma_{m-1}^{1}$. Supposons donc que $n>0$. On distingue deux cas :

i) $f_{n}$ est une application constante de valeur $\varepsilon=0,1$. L'application

$$
f^{\prime}: \square_{m} \longrightarrow \square_{n-1}, \quad \text { définie par } f^{\prime}=\left(f_{1}, \ldots, f_{n-1}\right),
$$

satisfait encore aux conditions $(a)$ et $(b)$, et par hypothèse de récurrence elle est donc un morphisme de $\boldsymbol{\square}^{\mathrm{c}}$. Or, on a l'égalité $f=f^{\prime} \otimes \delta_{1}^{1, \varepsilon}$, ce qui prouve l'assertion dans ce cas.

ii) $f_{n}=\sup _{E}$, où $E$ est une partie non vide de $\{1, \ldots, m\}$. On distingue deux cas : $\left.i i_{1}\right) n=1$. Alors $f=\sup _{E}$, et si $r=\operatorname{card}(E)$ et

$$
\{1, \ldots, m\}-E=\left\{i_{1}, \ldots, i_{m-r}\right\}
$$

avec $i_{1}<\cdots<i_{m-r}$, on a l'égalité

$$
f=\gamma_{1}^{1} \gamma_{2}^{1} \cdots \gamma_{r-1}^{1} \sigma_{r}^{i_{1}} \sigma_{r+1}^{i_{2}} \cdots \sigma_{m-1}^{i_{m-r}},
$$

ce qui prouve l'assertion dans ce cas.

ii $\left.2_{2}\right) n \geqslant 2$. Posons $q=\min (E)-1$. Alors l'application

$$
\square_{m} \longrightarrow \square_{n-1}, \quad \text { définie par }\left(f_{1}, \ldots, f_{n-1}\right),
$$

ne dépend que des $q$ premières coordonnées, et induit une application

$$
f^{\prime}: \square_{q} \longrightarrow \square_{n-1}
$$

qui satisfait encore aux conditions $(a)$ et $(b)$, et par hypothèse de récurrence est donc un morphisme de $\boldsymbol{\square}^{\mathrm{c}}$. D'autre part, si l'on pose

$$
E^{\prime}=\{i-q \mid i \in E\} \subset\{1, \ldots, m-q\}, \quad \text { et } \quad f^{\prime \prime}=\sup _{E^{\prime}}: \square_{m-q} \longrightarrow \square_{1},
$$

en vertu du cas $\left(i i_{1}\right)$, l'application $f^{\prime \prime}$ est un morphisme de $\mathbb{\square}^{\mathrm{c}}$. Or, on a l'égalité $f=f^{\prime} \otimes f^{\prime \prime}$, ce qui achève la démonstration.

Corollaire 2.4. Soient $m$ et $n$ deux entiers positifs ou nuls. L'application qui associe à un quadruplet $\left(F_{0}, F_{1}, F,\left(E_{j}\right)_{j \in F}\right)$, où $\left(F_{0}, F_{1}, F\right)$ est une partition de l'ensemble 
$\{1, \ldots, n\}$ (par des parties non nécessairement non vides), et $\left(E_{j}\right)_{j \in F}$ une famille de parties non vides de $\{1, \ldots, m\}$ telle que pour tous $j_{1}, j_{2} \in F$, si $j_{1}<j_{2}$, alors $\max \left(E_{j_{1}}\right)<\min \left(E_{j_{2}}\right)$, l'application

$$
f: \square_{m} \longrightarrow \square_{n}, \quad f=\left(f_{1}, \ldots, f_{n}\right), \quad f_{j}: \square_{m} \longrightarrow \square_{1}, \quad 1 \leqslant j \leqslant n,
$$

définie par

$$
f_{j}= \begin{cases}0, & \text { si } j \in F_{0}, \\ 1, & \text { si } j \in F_{1}, \\ \sup _{E_{j}}, & \text { si } j \in F,\end{cases}
$$

établit une bijection de l'ensemble de ces quadruplets sur l'ensemble $\operatorname{Hom}_{\square^{\mathrm{c}}}\left(\square_{m}, \square_{n}\right)$.

\section{Rappels sur les catégories test}

3.1. On note $\mathcal{C}$ at la catégorie des petites catégories et $\mathcal{W}$ la classe des équivalences faibles usuelles de $\mathcal{C}$ at, classe des foncteurs entre petites catégories dont l'image par le foncteur nerf est une équivalence faible simpliciale, autrement dit, un morphisme d'ensembles simpliciaux dont la réalisation topologique est une équivalence d'homotopie. On rappelle que la catégorie localisée $\mathrm{Hot}=\mathcal{W}^{-1} \mathcal{C}$ at est canoniquement équivalente à la catégorie homotopique des $\mathrm{CW}$-complexes. On vérifie aussitôt que la classe $\mathcal{W}$ est fortement saturée, autrement dit, si $\gamma: \mathcal{C} a t \longrightarrow$ Hot désigne le foncteur canonique de localisation, $\mathcal{W}$ est la classe des flèches de $\mathcal{C}$ at dont l'image par $\gamma$ est un isomorphisme. Si $u: A \longrightarrow B$ est un morphisme de $\mathcal{C} a t, u$ est une équivalence faible si et seulement si le foncteur opposé $u^{\circ}: A^{\circ} \longrightarrow B^{\circ}$ l'est, puisque les réalisations topologiques du nerf de $u$ et de $u^{\circ}$ coïncident. On dit qu'une petite catégorie $A$ est asphérique si l'unique foncteur $A \longrightarrow e$, où $e$ désigne la catégorie ponctuelle, est une équivalence faible, ou de façon équivalente, si $\gamma(A)$ est un objet final de Hot. Si une petite catégorie admet un objet final, ou initial, elle est asphérique. Pour tout foncteur entre petites catégories $u: A \longrightarrow B$ et tout objet $b$ de $B$, on note $A / b$ (resp. $b \backslash A$ ) la 《comma » catégorie dont les objets sont les couples $(a, s)$, où $a$ est un objet de $A$ et $s: u(a) \longrightarrow b$ (resp. $s: b \longrightarrow u(a))$ une flèche de $B$, un morphisme de $(a, s)$ vers un autre objet $\left(a^{\prime}, s^{\prime}\right)$ étant une flèche $f: a \longrightarrow a^{\prime}$ de $A$ telle que $s=s^{\prime} u(f)$ (resp. $\left.s^{\prime}=u(f) s\right)$. On dit que le foncteur $A \longrightarrow B$ est asphérique (resp. coasphérique) si pour tout objet $b$ de $B$, la catégorie $A / b$ (resp. $b \backslash A$ ) est asphérique. Il résulte du théorème A de Quillen [23] que tout foncteur asphérique ou coasphérique est une équivalence faible.

3.2. Dans la suite de cette section, on se fixe une petite catégorie $A$. On note $\widehat{A}$ la catégorie des préfaisceaux sur $A$. On rappelle qu'on a un couple de foncteurs adjoints

$$
\begin{array}{rlrl}
i_{A}: \widehat{A} & \longrightarrow \mathcal{C} a t, & i_{A}^{*}: \mathcal{C} a t & \longrightarrow \widehat{A} \\
X & \longmapsto A / X, \quad C \longmapsto\left(a \longmapsto \operatorname{Hom}_{\mathcal{C} a t}(A / a, C)\right) .
\end{array}
$$

On dit qu'un préfaisceau $X$ sur $A$ est asphérique si la catégorie $i_{A}(X)=A / X$ est asphérique. Si le préfaisceau $X$ est représentable, il est asphérique, puisque alors la catégorie $A / X$ admet un objet final. On dit qu'un morphisme de préfaisceaux sur $A$ est une équivalence faible si son image par le foncteur $i_{A}$ est une équivalence faible de 
$\mathcal{C} a t$. On note $\mathcal{W}_{\widehat{A}}$ la classe des équivalences faibles de $\widehat{A}$, de sorte que $\mathcal{W}_{\widehat{A}}=i_{A}^{-1}(\mathcal{W})$. La saturation forte de $\mathcal{W}$ implique alors celle de la classe $\mathcal{W}_{\widehat{A}}$. Si $A$ est la catégorie $\boldsymbol{\Delta}$ des simplexes, de sorte que $\widehat{A}$ soit la catégorie des ensembles simpliciaux, $\mathcal{W}_{\widehat{A}}$ est formée exactement des équivalences faibles simpliciales [16, chapitre 6 , section 3].

3.3. On dit que $A$ est une catégorie test faible si $\mathcal{W}=\left(i_{A}^{*}\right)^{-1}\left(\mathcal{W}_{\widehat{A}}\right)$, et si les foncteurs $i_{A}$ et $i_{A}^{*}$ induisent des équivalences de catégories

$$
\overline{i_{A}}: \mathcal{W}_{\widehat{A}}^{-1} \widehat{A} \longrightarrow \mathcal{W}^{-1} \mathcal{C} a t=\mathrm{Hot}, \quad \overline{i_{A}^{*}}: \text { Hot }=\mathcal{W}^{-1} \mathcal{C} a t \longrightarrow \mathcal{W}_{\widehat{A}}^{-1} \widehat{A}
$$

quasi-inverses l'une de l'autre. En particulier, les préfaisceaux sur $A$ sont alors des modèles pour les types d'homotopie. On dit que $A$ est une catégorie test locale si pour tout objet $a$ de $A$, la catégorie $A / a$ est une catégorie test faible. On dit que $A$ est une catégorie test si elle est à la fois une catégorie test locale et une catégorie test faible. On dit que $A$ est une catégorie test stricte si elle est une catégorie test et si le foncteur canonique de localisation $\widehat{A} \longrightarrow \mathcal{W}_{\widehat{A}}^{-1} \widehat{A} \simeq$ Hot commute aux produits binaires. L'exemple le plus important de catégorie test stricte est celui de la catégorie $\boldsymbol{\Delta}$ des simplexes [12, section 36], ou [22, proposition 1.6.13]. Un autre exemple est celui de la catégorie $\boldsymbol{\Theta}$ de Joyal $[\mathbf{3}, \mathbf{9}, \mathbf{1 9}]$.

3.4. On dit que la catégorie $A$ est totalement asphérique si elle est non vide, et si tout produit binaire dans $\widehat{A}$ dont les facteurs sont des préfaisceaux représentables est un préfaisceau asphérique. Une catégorie totalement asphérique est asphérique. Si $A$ est totalement asphérique, alors le produit de deux équivalences faibles de $\widehat{A}$ est une équivalence faible [22, propositions 1.6.1 et 2.1.3].

3.5. Un segment de $\widehat{A}$ est un triplet $\left(I, d^{0}, d^{1}\right)$, où $I$ est un préfaisceau sur $A$, et $d^{0}, d^{1}: e_{\widehat{A}} \longrightarrow I$ des morphismes de préfaisceaux, de l'objet final $e_{\widehat{A}}$ de $\widehat{A}$ vers $I$. On dit que ce segment est séparant si l'égalisateur de la double flèche $\left(d^{0}, d^{1}\right)$ est le préfaisceau vide. On dit qu'il est asphérique si le préfaisceau $I$ est asphérique.

Théorème 3.6 (Grothendieck). Soit A une petite catégorie. Si A est totalement asphérique et s'il existe un segment séparant asphérique de $\widehat{A}$, alors $A$ est une catégorie test stricte.

Voir [12, section 44, (c)], ou [22, proposition 1.6.8].

\section{La catégorie cubique avec connexions est une catégorie test stricte}

Lemme 4.1. Soient $m$ et $n$ deux entiers, $m, n \geqslant 0$. Le morphisme de préfaisceaux sur $\square$

$$
p=\mathrm{id}_{\square_{m}} \times \sigma_{n}^{1}: \square_{m} \times \square_{n+1} \longrightarrow \square_{m} \times \square_{n}
$$

est une équivalence faible de $\widehat{\mathbf{D}}^{\mathrm{c}}$. 
LA CATÉGORIE CUBIQUE AVEC CONNEXIONS EST UNE CATÉGORIE TEST STRICTE 315

Démonstration. Considérons le morphisme de préfaisceaux sur $\square^{\mathrm{c}}$

$$
s=\mathrm{id}_{\square_{m}} \times \delta_{n+1}^{1,1}: \square_{m} \times \square_{n} \longrightarrow \square_{m} \times \square_{n+1}
$$

et les foncteurs

$$
A=\square^{\mathrm{c}} / \square_{m} \times \square_{n+1} \frac{p_{*}=i_{\mathbb{\square}^{\mathrm{c}}}(p)}{\underset{s_{*}=i_{\mathbb{\square}^{\mathrm{c}}}(s)}{\longleftarrow}} \mathbb{\square}^{\mathrm{c}} / \square_{m} \times \square_{n}=B,
$$

déduits de $p$ et $s$ respectivement. On va montrer que $p_{*}$ et $s_{*}$ sont des équivalences faibles de $\mathcal{C}$ at, ce qui prouvera le lemme. Comme on a l'égalité $p s=\mathrm{id}_{\square_{m} \times \square_{n}}$, et par suite aussi l'égalité $p_{*} s_{*}=\mathrm{id}_{B}$, il suffit, en vertu de la forte saturation de la classe $\mathcal{W}$ des équivalences faibles de $\mathcal{C}$ at, de montrer que le foncteur $P=s_{*} p_{*}: A \longrightarrow A$ est une équivalence faible. Pour cela, on va définir un foncteur $D: A \longrightarrow A$, et des morphismes de foncteurs

$$
P \stackrel{\alpha}{\longrightarrow} D \stackrel{\beta}{\longleftarrow} \mathrm{id}_{A},
$$

et l'assertion résultera d'un argument standard d'homotopie (voir par exemple [22, lemme 1.4.6, (b), et exemple 1.4.15]).

a) Définition du foncteur $D: A \rightarrow A$. Pour tout objet

$$
\begin{aligned}
\left(\square_{q}, \square_{q} \stackrel{(a, b)}{\longrightarrow} \square_{m} \times \square_{n+1}\right) & a=\left(a_{1}, \ldots, a_{m}\right) \\
& b=\left(b_{0}, \ldots, b_{n}\right)
\end{aligned}
$$

de $A$, on définit $D\left(\square_{q},(a, b)\right)$ par la formule

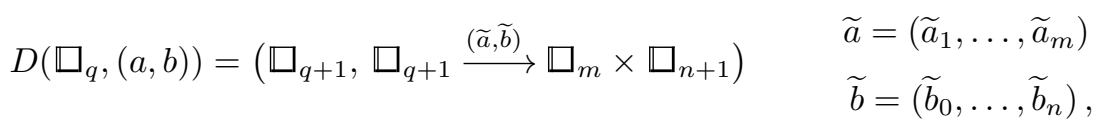

où pour tout $\left(x_{0}, x_{1}, \ldots, x_{q}\right) \in \square_{q+1}$,

$$
\begin{aligned}
& \widetilde{a}\left(x_{0}, x_{1}, \ldots, x_{q}\right)=a\left(x_{1}, \ldots, x_{q}\right), \\
& \widetilde{b}_{j}\left(x_{0}, x_{1}, \ldots, x_{q}\right)=\left\{\begin{array}{lc}
\sup \left\{x_{0}, b_{0}\left(x_{1}, \ldots, x_{q}\right)\right\}, & j=0, \\
b_{j}\left(x_{1}, \ldots, x_{q}\right), & 1 \leqslant j \leqslant n,
\end{array}\right.
\end{aligned}
$$

(l'application $\widetilde{b}$ ainsi définie étant un morphisme de $\square^{\mathrm{c}}$ en vertu, par exemple, de la proposition 2.3). Pour tout morphisme $f:\left(\square_{q},(a, b)\right) \longrightarrow\left(\square_{q^{\prime}},\left(a^{\prime}, b^{\prime}\right)\right)$ de $A$, on définit le morphisme $D(f):\left(\square_{q+1},(\widetilde{a}, \widetilde{b})\right) \longrightarrow\left(\square_{q^{\prime}+1},\left(\widetilde{a}^{\prime}, \widetilde{b}^{\prime}\right)\right)$ par la formule

$$
D(f)\left(x_{0}, x_{1}, \ldots, x_{q}\right)=\left(x_{0}, f\left(x_{1}, \ldots, x_{q}\right)\right) .
$$

On laisse au lecteur le soin de vérifier qu'on définit bien ainsi un endofoncteur de $A$.

b) Définition du morphisme de foncteurs $\alpha: P \rightarrow D$. On remarque que pour tout objet $\left(\square_{q},(a, b)\right)$ de $A$, on a par définition

$$
P\left(\square_{q},(a, b)\right)=\left(\square_{q}, s p \circ(a, b)\right)=\left(\square_{q},\left(a, \delta_{n+1}^{1,1} \sigma_{n}^{1} b\right)\right),
$$

et que si

$$
b=\left(b_{0}, b_{1}, \ldots, b_{n}\right): \square_{q} \longrightarrow \square_{n+1},
$$


on a

$$
\delta_{n+1}^{1,1} \sigma_{n}^{1} b=\left(1, b_{1}, \ldots, b_{n}\right) .
$$

Pour tout objet $\left(\square_{q},(a, b)\right)$ de $A$, on définit

$$
\alpha_{\left(\square_{q},(a, b)\right)}: P\left(\square_{q},(a, b)\right)=\left(\square_{q},\left(a, \delta_{n+1}^{1,1} \sigma_{n}^{1} b\right)\right) \longrightarrow\left(\square_{q+1},(\widetilde{a}, \widetilde{b})\right)=D\left(\square_{q},(a, b)\right)
$$

par la formule

$$
\alpha_{\left(\square_{q},(a, b)\right)}\left(x_{1}, \ldots, x_{q}\right)=\left(1, x_{1}, \ldots, x_{q}\right) .
$$

On vérifie facilement qu'on définit bien ainsi un morphisme de $A$, et que ce morphisme est fonctoriel en $\left(\square_{q},(a, b)\right)$.

c) Définition du morphisme de foncteurs $\beta: \mathrm{id}_{A} \rightarrow D$. Pour tout objet $\left(\square_{q},(a, b)\right)$ de $A$, on définit

$$
\beta_{\left(\square_{q},(a, b)\right)}:\left(\square_{q},(a, b)\right) \longrightarrow\left(\square_{q+1},(\widetilde{a}, \widetilde{b})\right)=D\left(\square_{q},(a, b)\right)
$$

par la formule

$$
\beta_{\left(\square_{q},(a, b)\right)}\left(x_{1}, \ldots, x_{q}\right)=\left(0, x_{1}, \ldots, x_{q}\right),
$$

et on constate aussitôt qu'on définit ainsi un morphisme de $A$, et que ce morphisme est fonctoriel en $\left(\square_{q},(a, b)\right)$.

Proposition 4.2. La catégorie $\square^{\mathrm{c}}$ est totalement asphérique.

Démonstration. En vertu de la stabilité des équivalences faibles par composition, le lemme précédent implique par une récurrence immédiate que pour tous $m, n \geqslant 0$, la projection $\square_{m} \times \square_{n} \longrightarrow \square_{m} \times \square_{0} \simeq \square_{m}$ est une équivalence faible de préfaisceaux sur $\square^{\mathrm{c}}$. Comme $\square_{m}$, étant représentable, est un préfaisceau asphérique, il en est de même de $\square_{m} \times \square_{n}$, ce qui prouve la proposition.

Proposition 4.3. La catégorie $\square^{\mathrm{c}}$ est une catégorie test stricte.

Démonstration. En vertu du théorème 3.6 et de la proposition précédente, il suffit de montrer l'existence d'un segment séparant asphérique dans $\widehat{\boldsymbol{D}}^{\mathrm{c}}$. Or, on vérifie immédiatement que $\left(\square_{1}, \delta_{1}^{1,0}, \delta_{1}^{1,1}\right)$ est un segment séparant de $\widehat{\boldsymbol{\square}}^{\mathrm{c}}$, et comme $\square_{1}$ est représentable, il est asphérique, ce qui prouve l'assertion.

\section{Cubes avec connexions et segments multiplicatifs au sens de Grothendieck}

5.1. Le but de cette section, un peu plus spéculative, est de faire le lien entre la théorie des ensembles cubiques avec connexions, et celle des segments multiplicatifs de Grothendieck.

5.2. Soit $(\mathcal{V}, \otimes, e)$ une catégorie monoïdale stricte, de produit tensoriel

$$
\otimes: \mathcal{V} \times \mathcal{V} \longrightarrow \mathcal{V}, \quad X, Y \longmapsto X \otimes Y,
$$

et d'objet unité $e$. Un cylindre dans $\mathcal{V}$ est un quadruplet $\left(I, d^{0}, d^{1}, p\right)$, où $I$ est un objet de $\mathcal{V}$, et $d^{0}, d^{1}: e \longrightarrow I, p: I \longrightarrow e$ des morphismes de $\mathcal{V}$ satisfaisant aux relations 
$p d^{0}=\mathrm{id}_{e}=p d^{1}$. Si $\mathcal{V}$ est la catégorie des endofoncteurs d'une catégorie $C$, munie de la structure monoïdale définie par la composition des endofoncteurs, alors un cylindre dans $\mathcal{V}$ est un cylindre au sens de $[\mathbf{2 0}]$. Si $\mathcal{V}$ est la catégorie des cubes $\boldsymbol{\square}$, munie de la structure monoïdale du paragraphe 2.2 , alors $\left(\square_{1}, \delta_{1}^{1,0}, \delta_{1}^{1,1}, \sigma_{0}^{1}\right)$ est un cylindre de $\square$.

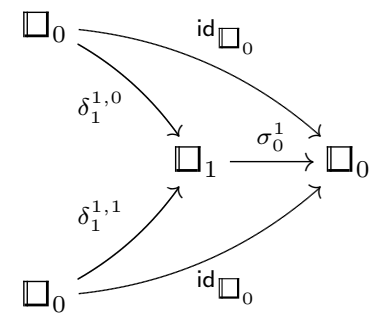

En fait, cet exemple est « universel», au sens de la proposition suivante qui est une variante de [11, théorème 4.2] ou de [8, proposition 8.4.6].

Proposition 5.3. Soit $(\mathcal{V}, \otimes, e)$ une catégorie monö̈dale stricte. L'application associant à un foncteur $F: \square \longrightarrow \mathcal{V}$ le quadruplet

$$
\left(F\left(\square_{1}\right), F\left(\delta_{1}^{1,0}\right), F\left(\delta_{1}^{1,1}\right), F\left(\sigma_{0}^{1}\right)\right)
$$

établit une bijection entre l'ensemble des foncteurs monoïdaux stricts de $\square$ vers $\mathcal{V}$ et celui des cylindres de $\mathcal{V}$.

5.4. Soit $(\mathcal{V}, \otimes, e)$ une catégorie monoïdale stricte. Un cylindre multiplicatif dans $\mathcal{V}$ est un cylindre $\left(I, d^{0}, d^{1}, p\right)$ de $\mathcal{V}$, muni d'un morphisme $m: I \otimes I \longrightarrow I$ satisfaisant aux relations suivantes :

$$
\begin{gathered}
m\left(m \otimes \mathrm{id}_{I}\right)=m\left(\mathrm{id}_{I} \otimes m\right), \\
m\left(d^{0} \otimes \mathrm{id}_{I}\right)=\mathrm{id}_{I}=m\left(\mathrm{id}_{I} \otimes d^{0}\right), \\
m\left(d^{1} \otimes \mathrm{id}_{I}\right)=d^{1} p=m\left(\mathrm{id}_{I} \otimes d^{1}\right), \\
p m=p \otimes p .
\end{gathered}
$$

La relation 5.4.1 exprime que $m$ est une « loi de composition » associative, et la relation 5.4 .2 que $d^{0}: e \rightarrow I$ est une « unité » pour cette loi de composition, de sorte que le triplet $\left(I, m, d^{0}\right)$ est un «monoïde » de $\mathcal{V}$. La relation 5.4 .4 exprime que $p: I \longrightarrow e$ est un morphisme de monoïdes, l'objet $e$ étant muni de la structure triviale de monoïde $\left(e, \mathrm{id}_{e}, \mathrm{id}_{e}\right)$. Enfin, la relation 5.4.3 exprime que $d^{1}: e \longrightarrow I$ est un « zéro » ou « élément absorbant » pour la loi de composition $m$. La notion de cylindre multiplicatif est proche de celle de « monoïde cubique» introduite par M. Grandis [10], avec la différence qu'un cylindre multiplicatif comporte une seule « multiplication », au lieu de deux pour un monoïde cubique.

Si $\mathcal{V}$ est la catégorie des cubes avec connexions $\square^{\mathrm{c}}$, munie de la structure monoïdale du paragraphe 2.2 , alors le cylindre $\left(\square_{1}, \delta_{1}^{1,0}, \delta_{1}^{1,1}, \sigma_{0}^{1}\right)$, muni du morphisme

$$
\gamma_{1}^{1}: \square_{2}=\square_{1} \otimes \square_{1} \longrightarrow \square_{1},
$$

est un cylindre multiplicatif de $\mathbb{\square}^{\mathrm{c}}$. Cet exemple est « universel » au sens de la proposition suivante, analogue à la proposition 5.3 (et très proche de [11, théorème 5.2$]$ ). 
Proposition 5.5. Soit $(\mathcal{V}, \otimes, e)$ une catégorie monö̈dale stricte. L'application associant à un foncteur $F: \square^{\mathrm{c}} \longrightarrow \mathcal{V}$ le quintuplet

$$
\left(F\left(\square_{1}\right), F\left(\delta_{1}^{1,0}\right), F\left(\delta_{1}^{1,1}\right), F\left(\sigma_{0}^{1}\right), F\left(\gamma_{1}^{1}\right)\right)
$$

établit une bijection entre l'ensemble des foncteurs monö̈daux stricts de $\square^{\mathrm{c}}$ vers $\mathcal{V}$ et celui des cylindres multiplicatifs de $\mathcal{V}$.

5.6. Dans ce qui suit, on esquisse une preuve de cette proposition. Pour cela, on rappelle que les générateurs

$$
\begin{array}{ll}
\delta_{n}^{i, \varepsilon}: \square_{n-1} \longrightarrow \square_{n}, & n \geqslant 1, \quad 1 \leqslant i \leqslant n, \quad \varepsilon=0,1, \\
\sigma_{n}^{i}: \square_{n+1} \longrightarrow \square_{n}, & n \geqslant 0, \quad 1 \leqslant i \leqslant n+1, \\
\gamma_{n}^{i}: \square_{n+1} \longrightarrow \square_{n}, & n \geqslant 1, \quad 1 \leqslant i \leqslant n,
\end{array}
$$

de $\square^{\mathrm{c}}$ satisfont aux relations suivantes (où $\varepsilon, \eta \in\{0,1\}$ )

$$
\begin{aligned}
& \delta_{n}^{j, \eta} \delta_{n-1}^{i, \varepsilon}=\delta_{n}^{i, \varepsilon} \delta_{n-1}^{j-1, \eta}, \quad i<j, \\
& \sigma_{n}^{j} \sigma_{n+1}^{i}=\sigma_{n}^{i} \sigma_{n+1}^{j+1}, \quad i \leqslant j, \\
& \sigma_{n}^{j} \delta_{n+1}^{i, \varepsilon}= \begin{cases}\delta_{n}^{i, \varepsilon} \sigma_{n-1}^{j-1}, & i<j, \\
\operatorname{id}_{\square_{n}}, & i=j, \\
\delta_{n}^{i-1, \varepsilon} \sigma_{n-1}^{j}, & i>j,\end{cases} \\
& \gamma_{n}^{j} \gamma_{n+1}^{i}=\gamma_{n}^{i} \gamma_{n+1}^{j+1}, \quad i \leqslant j, \\
& \gamma_{n}^{j} \delta_{n+1}^{i, \varepsilon}= \begin{cases}\delta_{n}^{i, \varepsilon} \gamma_{n-1}^{j-1}, & i<j, \\
\operatorname{id}_{\square_{n}}, & i=j, j+1, \quad \varepsilon=0, \quad l=1, j+1, \quad \varepsilon=1, \\
\delta_{n}^{j, 1} \sigma_{n-1}^{j}, & i>j+1, \\
\delta_{n}^{i-1, \varepsilon} \gamma_{n-1}^{j}, & \end{cases} \\
& \sigma_{n}^{j} \gamma_{n+1}^{i}= \begin{cases}\gamma_{n}^{i} \sigma_{n+1}^{j+1}, & i<j, \\
\sigma_{n}^{i} \sigma_{n+1}^{i}, & i=j, \\
\gamma_{n}^{i-1} \sigma_{n+1}^{j}, & i>j,\end{cases}
\end{aligned}
$$

et qu'on obtient ainsi une présentation de $\mathbb{\square}^{\mathrm{c}}$ par générateurs et relations. On pose

$$
\begin{aligned}
& \delta^{0}=\delta_{1}^{1,0}, \delta^{1}=\delta_{1}^{1,1}: e=\square_{0} \longrightarrow \square_{1}, \\
& \sigma=\sigma_{0}^{1}: \square_{1} \longrightarrow \square_{0}=e, \\
& \gamma=\gamma_{1}^{1}: \square_{1} \otimes \square_{1}=\square_{2} \longrightarrow \square_{1},
\end{aligned}
$$

et on remarque que les relations 5.6.1 et 5.6.2 impliquent les égalités

$$
\begin{gathered}
\sigma \delta^{0}=\mathrm{id}_{\square_{0}}=\sigma \delta^{1}, \\
\gamma\left(\gamma \otimes \mathrm{id}_{\square_{1}}\right)=\gamma\left(\mathrm{id}_{\square_{1}} \otimes \gamma\right),
\end{gathered}
$$


LA CATÉGORIE CUBIQUE AVEC CONNEXIONS EST UNE CATÉGORIE TEST STRICTE 319

$$
\begin{gathered}
\gamma\left(\delta^{0} \otimes \mathrm{id}_{\square_{1}}\right)=\mathrm{id}_{\square_{1}}=\gamma\left(\mathrm{id}_{\square_{1}} \otimes \delta^{0}\right), \\
\gamma\left(\delta^{1} \otimes \mathrm{id}_{\square_{1}}\right)=\delta^{1} \sigma=\gamma\left(\mathrm{id}_{\square_{1}} \otimes \delta^{1}\right), \\
\sigma \gamma=\sigma \otimes \sigma,
\end{gathered}
$$

exprimant que $\left(\square_{1}, \delta^{0}, \delta^{1}, \sigma, \gamma\right)$ est un cylindre multiplicatif. D'autre part, on remarque qu'on a les formules

$$
\begin{array}{ll}
\delta_{n}^{i, \varepsilon}=\mathrm{id}_{\square_{i-1}} \otimes \delta^{\varepsilon} \otimes \mathrm{id}_{\square_{n-i}}, & n \geqslant 1, \quad 1 \leqslant i \leqslant n, \quad \varepsilon=0,1, \\
\sigma_{n}^{i}=\mathrm{id}_{\square_{i-1}} \otimes \sigma \otimes \mathrm{id}_{\square_{n+1-i}}, & n \geqslant 0, \quad 1 \leqslant i \leqslant n+1, \\
\gamma_{n}^{i}=\mathrm{id}_{\square_{i-1}} \otimes \gamma \otimes \mathrm{id}_{\square_{n-i}}, & n \geqslant 1, \quad 1 \leqslant i \leqslant n .
\end{array}
$$

Une vérification longue et fastidieuse, mais sans aucune difficulté, montre, en utilisant ces formules et la structure de catégorie monoïdale de la catégorie $\mathbf{\square}^{\mathrm{c}}$, que réciproquement les relations 5.6.3-5.6.7 impliquent les relations 5.6.1 et 5.6.2. Vu que les relations 5.6.1-5.6.2 constituent une présentation de la catégorie $\mathbb{\square}^{\mathrm{c}}$, cela signifie exactement que la catégorie monoïdale $\left(\boldsymbol{\square}^{\mathrm{c}}, \otimes, e=\square_{0}\right)$ est la catégorie monoïdale stricte universelle engendrée par un objet $\square_{1}$ et les flèches $\delta^{0}, \delta^{1}, \sigma, \gamma$, assujetties aux relations 5.6.3-5.6.7, ce qui est simplement une reformulation de la proposition 5.5.

Remarque 5.7. Dans la théorie de l'homotopie de Grothendieck [12, 22], la notion de segment multiplicatif occupe une place importante. Si $\mathcal{M}$ est une catégorie admettant des produits finis, d'objet final noté $e$, un segment multiplicatif dans $\mathcal{M}$ est un segment $d^{0}, d^{1}: e \longrightarrow I$, muni d'une « loi de composition» $m: I \times I \longrightarrow I$ admettant $d^{0}$ comme « unité à gauche» et $d^{1}$ comme « zéro à gauche», autrement dit, telle que

$$
m\left(d^{0} \times \mathrm{id}_{I}\right)=\mathrm{id}_{I} \quad \text { et } \quad m\left(d^{1} \times \mathrm{id}_{I}\right)=d^{1} p,
$$

où $p: I \longrightarrow e$ désigne l'unique morphisme de $I$ vers $e$. Comme $e$ est un objet final, on remarque qu'on a automatiquement les relations

$$
p d^{0}=\mathrm{id}_{e}=p d^{1} \quad \text { et } \quad p m=p \times p .
$$

Ainsi, si on considère $\mathcal{M}$ comme une catégorie monoïdale (en général non stricte; mais cela est sans conséquence pour notre propos) avec comme produit tensoriel $\otimes$ le produit catégorique $\times$, et comme objet unité l'objet final $e$, les relations 5.7.1 et 5.7.2 font partie des relations définissant un cylindre multiplicatif de $\mathcal{M}$. Plus précisément, dans le cas d'un cylindre multiplicatif, on demande de plus que la loi de composition $m$ soit associative, et que $d^{0}$ (resp. $d^{1}$ ) soit non seulement une unité (resp. un zéro) à gauche, mais aussi à droite. Il est à noter que dans la plupart des exemples de segments multiplicatifs étudiés par Grothendieck (mais pas dans tous), ses relations supplémentaires sont satisfaites. En particulier, c'est bien le cas dans l'exemple le plus important qu'il considère, celui de l'objet de Lawvere classifiant les sous-objets dans un topos, où les sections $d^{0}$ et $d^{1}$ sont définies par les sous-objets plein et vide respectivement, et la loi de composition $m$ par l'intersection de deux sous-objets. L'objet de Lawvere est même muni d'une structure de monoïde cubique au sens de $[\mathbf{1 0}]$, la deuxième loi de composition étant définie par la réunion de deux sous-objets. 
Le fait que la catégorie $\mathbb{\square}^{c}$ des cubes avec connexions soit la catégorie monoïdale stricte universelle abritant un cylindre multiplicatif explique peut-être ses propriétés homotopiques remarquables. La théorie des segments multiplicatifs de Grothendieck suggère aussi qu'une partie des relations définissant cette catégorie n'est pas nécessaire pour démontrer qu'elle est une catégorie test stricte. On laisse le soin au lecteur de vérifier qu'en effet les preuves de la section 4 permettent de prouver que si l'on appelle cylindre multiplicatif faible dans une catégorie monoïdale stricte $(\mathcal{V}, \otimes, e)$, un cylindre $\left(I, d^{0}, d^{1}, p\right)$ dans $\mathcal{V}$, muni d'une loi de composition $m: I \otimes I \longrightarrow I$ telle que

$$
m\left(d^{0} \otimes \mathrm{id}_{I}\right)=\mathrm{id}_{I}, \quad m\left(d^{1} \otimes \mathrm{id}_{I}\right)=d^{1} p, \quad \text { et } \quad p m=p \otimes p,
$$

alors la catégorie monoïdale stricte universelle abritant un tel cylindre est une catégorie test stricte. Il suffit pour cela de remarquer que dans une telle catégorie monoïdale universelle l'objet unité est aussi un objet final, et traduire les formules de la démonstration du lemme 4.1, en termes des générateurs.

\section{La catégorie cubique classique n'est pas une catégorie test stricte}

6.1. Le but de cette section est de montrer que la catégorie cubique classique (sans connexions) $\mathbb{\square}$, bien qu'elle soit une catégorie test [8, corollaire 8.4.13, ou proposition 8.4.27], n'est pas une catégorie test stricte. Pour cela, on va montrer qu'elle n'est pas totalement asphérique, et plus précisément que le préfaisceau $\square_{1} \times \square_{1}$ sur $\square$, ou de façon équivalente la catégorie $\square / \square_{1} \times \square_{1}$, a le type d'homotopie d'une sphère $S^{2}$ munie d'une corde, autrement dit du bouquet $S^{2} \vee S^{1}$, et en particulier n'est pas asphérique.

6.2. Notons $A$ la sous-catégorie pleine de $\square / \square_{1} \times \square_{1}$ formée des onze objets suivants, où $\varepsilon, \eta \in\{0,1\}$ :

$$
\begin{aligned}
& h_{+}=\left(\square_{2}, \square_{2} \stackrel{\left(p r_{1}, p r_{2}\right)=\left(\sigma_{1}^{2}, \sigma_{1}^{1}\right)}{\longrightarrow} \square_{1} \times \square_{1}\right), \\
& h_{-}=\left(\square_{2}, \square_{2} \stackrel{\left(p r_{2}, p r_{1}\right)=\left(\sigma_{1}^{1}, \sigma_{1}^{2}\right)}{\longrightarrow} \square_{1} \times \square_{1}\right), \\
& (\mathrm{id}, \varepsilon)=\left(\square_{1}, \square_{1} \stackrel{\left.{ }^{(\mathrm{id}} \square_{1}, \delta_{1}^{1, \varepsilon} \sigma_{0}^{1}\right)}{\longrightarrow} \square_{1} \times \square_{1}\right), \\
& (\varepsilon, \text { id })=\left(\square_{1}, \square_{1} \stackrel{\left(\delta_{1}^{1, \varepsilon} \sigma_{0}^{1}, \text { id } \square_{1}\right)}{\longrightarrow} \square_{1} \times \square_{1}\right), \\
& c=\left(\square_{1}, \square_{1} \stackrel{\left({ }^{\left(d_{\square_{1}}\right.}{ }_{1}^{, \text {id }} \square_{1}\right)}{\longrightarrow} \square_{1} \times \square_{1}\right), \\
& (\varepsilon, \eta)=\left(\square_{0}, \square_{0} \stackrel{\left(\delta_{1}^{1, \varepsilon}, \delta_{1}^{1, \eta}\right)}{\longrightarrow} \square_{1} \times \square_{1}\right) .
\end{aligned}
$$

Cette catégorie est en fait un ensemble ordonné, et cet ensemble ordonné est celui des strates fermées, ordonnées par inclusion, d'un espace $X$ formé d'une sphère $S^{2}$ 
munie d'une corde $c$, stratifié comme suit.

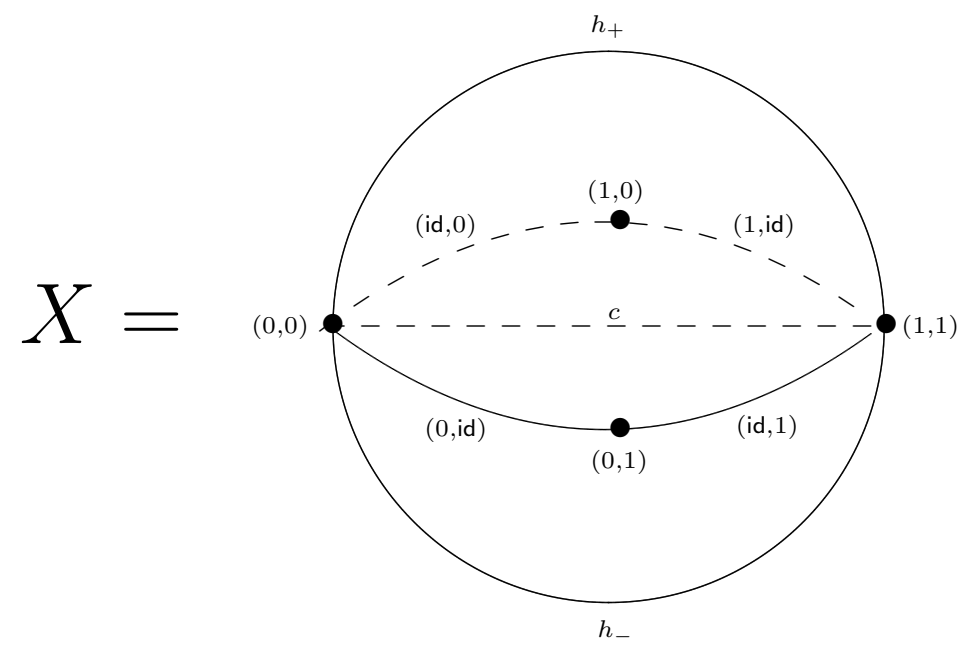

Il y a deux strates de dimension 2 , l'hémisphère supérieur $h_{+}$et l'hémisphère inférieur $h_{-}$, cinq strates de dimension 1 , la corde $c$ et quatre segments formant l'équateur de la sphère, et quatre strates de dimension 0 délimitant ces quatre segments, dont deux n'appartenant pas à un même segment sont les extrémités de la corde. Cette stratification définit un foncteur $F$ de $A$ vers la catégorie des espaces topologiques, qui est cofibrant pour la structure de catégorie de modèles de Reedy sur cette catégorie de foncteurs $[\mathbf{1 4}, \mathbf{1 5}], A$ étant considérée munie de l'application dimension

$$
(\varepsilon, \eta) \longmapsto 0, \quad(\mathrm{id}, \varepsilon),(\varepsilon, \mathrm{id}), c \longmapsto 1, \quad h_{+}, h_{-} \longmapsto 2,
$$

définie par la dimension des strates correspondantes de $X$. On a donc des isomorphismes dans la catégorie homotopique

$$
\underset{A}{\underset{\operatorname{holim}}{\longrightarrow}} F \simeq \underset{A}{\lim } F \simeq X,
$$

et comme le foncteur $F$ est à valeurs des espaces contractiles, $\underline{\text { holim }}_{A} F$ a le type d'homotopie de la catégorie $A$. On en déduit que $A$ a le type d'homotopie d'une sphère $S^{2}$ munie d'une corde. Pour montrer qu'il en est de même de la catégorie $\square / \square_{1} \times \square_{1}$, il suffit de montrer que le foncteur d'inclusion $A \longrightarrow \square / \square_{1} \times \square_{1}$ est une équivalence faible de $\mathcal{C}$ at. Cela résulte du lemme suivant :

Lemme 6.3. Le foncteur d'inclusion $A \longrightarrow \square / \square_{1} \times \square_{1}$ est un foncteur coasphérique.

Démonstration. Soit $\left(\square_{q}, \varphi: \square_{q} \rightarrow \square_{1} \times \square_{1}\right)$ un objet de $\square / \square_{1} \times \square_{1}$. Il s'agit de montrer que la catégorie $C=\left(\square_{q}, \varphi\right) \backslash A$ est asphérique, et pour cela, on va montrer qu'elle admet un objet initial. On distingue plusieurs cas : 
a) si $\varphi=\left(p r_{i}, p r_{j}\right): \square_{q} \longrightarrow \square_{1} \times \square_{1}$ et $1 \leqslant i<j \leqslant q$, alors $C$ admet

$$
\left(h_{+},\left(p r_{i}, p r_{j}\right):\left(\square_{q}, \varphi\right) \longrightarrow h_{+}\right)
$$

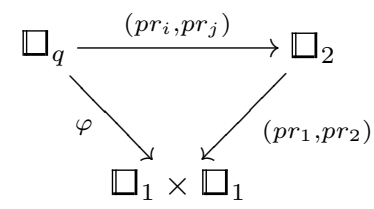

comme objet initial (et elle est même réduite à cet objet initial);

b) si $\varphi=\left(p r_{i}, p r_{j}\right): \square_{q} \longrightarrow \square_{1} \times \square_{1}$ et $1 \leqslant j<i \leqslant q$, alors $C$ admet

$$
\left(h_{-},\left(p r_{j}, p r_{i}\right):\left(\square_{q}, \varphi\right) \longrightarrow h_{-}\right)
$$

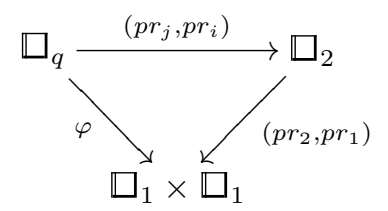

comme objet initial (et elle est même réduite à cet objet initial);

c) si $\varphi=\left(p r_{i}, p r_{i}\right): \square_{q} \longrightarrow \square_{1} \times \square_{1}, 1 \leqslant i \leqslant q$, alors $C$ admet

$$
\left(c, p r_{i}:\left(\square_{q}, \varphi\right) \longrightarrow c\right)
$$

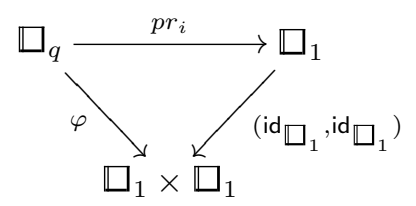

comme objet initial;

d) si $\varphi=\left(p r_{i}, \varepsilon\right): \square_{q} \longrightarrow \square_{1} \times \square_{1}, 1 \leqslant i \leqslant q, \varepsilon=0,1$, alors $C$ admet

$$
\left((\mathrm{id}, \varepsilon), p r_{i}:\left(\square_{q}, \varphi\right) \longrightarrow(\mathrm{id}, \varepsilon)\right)
$$

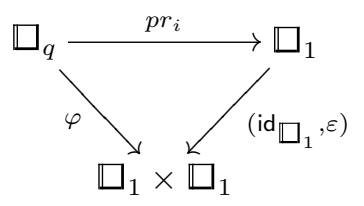

comme objet initial ;

e) si $\varphi=\left(\varepsilon, p r_{i}\right): \square_{q} \longrightarrow \square_{1} \times \square_{1}, 1 \leqslant i \leqslant q, \varepsilon=0,1$, alors $C$ admet

$$
\left((\varepsilon, \mathrm{id}), p r_{i}:\left(\square_{q}, \varphi\right) \longrightarrow(\varepsilon, \mathrm{id})\right)
$$

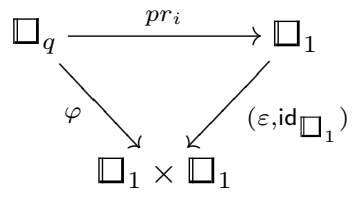

comme objet initial ;

f) si $\varphi=(\varepsilon, \eta): \square_{q} \longrightarrow \square_{1} \times \square_{1}, \varepsilon, \eta=0,1$, alors $C$ admet

$$
\left((\varepsilon, \eta),\left(\square_{q}, \varphi\right) \longrightarrow(\varepsilon, \eta)\right)
$$

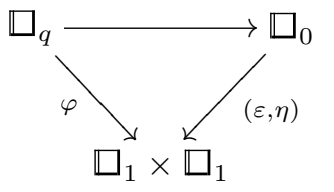

comme objet initial. 
Remarque 6.4. On a tenu à présenter ci-dessus une preuve entièrement élémentaire de ce lemme. Il peut néanmoins se démontrer de façon plus conceptuelle en utilisant la théorie des catégories squelettiques régulières de D.-C. Cisinski [8, chapitre 8]. Une catégorie squelettique régulière est une petite catégorie $B$, munie d'une fonction dimension $\lambda$ : Ob $B \rightarrow \mathbb{N}$, telle que si l'on note $B_{+}$(resp. $B_{-}$) l'ensemble des monomorphismes (resp. des épimorphismes scindés) de $B$, les conditions suivantes soient satisfaites (voir loc. cit., définitions 8.1.1, 8.1.36, 8.2.3, remarque 8.1.2, et propositions 8.1.37, 8.2.2) :

(a) si $b \longrightarrow b^{\prime}$ est une flèche non identique de $B$ appartenant à $B_{+}$(resp. à $B_{-}$), alors $\lambda(b)<\lambda\left(b^{\prime}\right)\left(\right.$ resp. $\left.\lambda(b)>\lambda\left(b^{\prime}\right)\right)$;

(b) toute flèche de $B$ se décompose de façon unique en une flèche de $B_{-}$suivie d'une flèche de $B_{+}$;

(c) si deux épimorphismes scindés de $B$ ont même ensemble de sections, ils sont égaux.

Si $X$ est un préfaisceau sur $B, b$ un objet de $B$, et $x: b \longrightarrow X$ une section de $X$ audessus de $b$, on dit que la section $x$ de $X$ est dégénérée s'il existe un épimorphisme scindé non identique $p: b \longrightarrow b^{\prime}$ de $B$, et une section $x^{\prime}: b^{\prime} \longrightarrow X$ tels que $x=x^{\prime} p$. On dit que la section $x$ est non dégénérée si elle n'est pas dégénérée. Il est facile de vérifier que si le morphisme de préfaisceaux $x: b \longrightarrow X$ est un monomorphisme, alors la section $x$ est non dégénérée. Si réciproquement, pour tout objet $b$ de $B$, toute section non dégénérée $b \longrightarrow X$ de $X$ au-dessus de $b$ est un monomorphisme de préfaisceaux, on dit que le préfaisceau $X$ sur $B$ est régulier. Si $B$ est une catégorie squelettique régulière, on a un « lemme d'Eilenberg-Zilber» qui se formule dans ce cas comme suit (voir loc. cit., corollaire 8.1.14 et proposition 8.1.24) : pour tout préfaisceau $X$ sur $B$, tout objet $b$ de $B$, et toute section $x: b \longrightarrow X$ de $X$ au-dessus de $b$, il existe un couple unique $\left(p, x^{\prime}\right)$, où $p: b \longrightarrow b^{\prime}$ est un épimorphisme scindé de $B$, et $x^{\prime}: b^{\prime} \longrightarrow X$ une section non dégénérée de $X$, tels que $x=x^{\prime} p$. Le couple $\left(p, x^{\prime}\right)$ s'appelle alors la décomposition d'Eilenberg-Zilber de $x$.

La catégorie des cubes $\square$, munie de l'application dimension $\lambda: \square_{m} \mapsto m$, est un exemple de catégorie squelettique régulière (loc. cit., proposition 8.4.17), et il est facile de vérifier que pour tous $m, n \geqslant 0$, le préfaisceau produit $\square_{m} \times \square_{n}$ est régulier. En particulier $\square_{1} \times \square_{1}$ est un préfaisceau régulier sur $\square$, et en gardant les notations du paragraphe 6.2 , on remarque que $A$ est la sous-catégorie pleine de $\square / \square_{1} \times \square_{1}$ formée des objets $\left(\square_{q}, \varphi: \square_{q} \longrightarrow \square_{1} \times \square_{1}\right)$ tels que la section $\varphi$ du préfaisceau $\square_{1} \times \square_{1}$ au-dessus de l'objet $\square_{q}$ de $\square$ soit non dégénérée. Ainsi le lemme suivant, bien utile pour calculer le type d'homotopie d'un préfaisceau régulier, généralise et explique le lemme 6.3.

Lemme 6.5. Soient $B$ une catégorie squelettique régulière, $X$ un préfaisceau régulier sur $B$, et $A$ la sous-catégorie pleine de $B / X$ formée des objets $(b, x: b \longrightarrow X)$ tels que $x$ soit une section non dégénérée de $X$ au-dessus de $b$. Alors le foncteur d'inclusion $A \longrightarrow B / X$ est coasphérique.

Démonstration. Il s'agit de prouver que pour tout objet $(b, x: b \rightarrow X)$ de $B / X$, la catégorie $(b, x) \backslash A$ est asphérique, et pour cela il suffit de prouver qu'elle admet un objet initial. Soit $\left(p: b \longrightarrow b^{\prime}, x^{\prime}: b^{\prime} \longrightarrow X\right)$ la décomposition d'Eilenberg-Zilber de la 
section $x$ de $X$. Comme la section $x^{\prime}$ de $X$ est non dégénérée, $\left(b^{\prime}, x^{\prime}: b^{\prime} \longrightarrow X\right)$ est un objet de $A$, et l'égalité $x=x^{\prime} p$ implique que $p$ induit un morphisme de $B / X$ de source $(b, x)$ et de but $\left(b^{\prime}, x^{\prime}\right)$, d'où un objet

$$
\left(\left(b^{\prime}, x^{\prime}\right), p:(b, x) \longrightarrow\left(b^{\prime}, x^{\prime}\right)\right)
$$

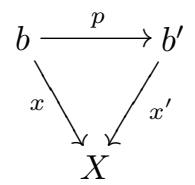

de $(b, x) \backslash A$. On va montrer que cet objet est un objet initial de $(b, x) \backslash A$. Soit donc

$$
((c, y), g:(b, x) \longrightarrow(c, y))
$$

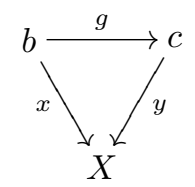

un objet arbitraire de $(b, x) \backslash A$. Comme le préfaisceau $X$ est régulier et la section $y$ non dégénérée, le morphisme de préfaisceaux $y: c \longrightarrow X$ est un monomorphisme et il existe au plus un morphisme $\left(b^{\prime}, x^{\prime}\right) \longrightarrow(c, y)$ de $A$, et à plus forte raison, au plus un morphisme

$$
\left(\left(b^{\prime}, x^{\prime}\right), p:(b, x) \longrightarrow\left(b^{\prime}, x^{\prime}\right)\right) \longrightarrow((c, y), g:(b, x) \longrightarrow(c, y))
$$

de $(b, x) \backslash A$. Il suffit donc de prouver qu'un tel morphisme existe. Soit

$$
g=s q, \quad b \stackrel{q}{\longrightarrow} c^{\prime} \stackrel{s}{\longrightarrow} c
$$

l'unique décomposition de $g$ en un épimorphisme scindé suivi d'un monomorphisme. Alors le morphisme de préfaisceaux $y s: c^{\prime} \longrightarrow X$ est un monomorphisme, et par suite $y s$ est une section non dégénérée de $X$ au-dessus de $c^{\prime}$, et le couple $(q, y s)$ est la décomposition d'Eilenberg-Zilber de la section $y g$ de $X$ au-dessus de $b$. Comme $y g=x$, l'unicité de la décomposition d'Eilenberg-Zilber montre que $c^{\prime}=b^{\prime}, q=p$, et $x^{\prime}=y s$, ce qui prouve que $s: b^{\prime} \longrightarrow c$ induit le morphisme cherché.

Remarque 6.6. Il résulte facilement de la proposition 2.3 que la catégorie $\boldsymbol{\square}^{\mathrm{c}}$ est aussi squelettique régulière. En revanche, le préfaisceau $\square_{1} \times \square_{1}$ sur $\mathbb{\square}^{\mathrm{c}}$ n'est pas régulier. Par exemple, on vérifie immédiatement que le morphisme $\varphi: \square_{3} \longrightarrow \square_{1} \times \square_{1}$ de $\widehat{\mathbf{D}}^{\mathrm{c}}$, où $\varphi=\left(\varphi_{1}, \varphi_{2}\right)$ est défini par les formules

$$
\varphi_{1}\left(x_{1}, x_{2}, x_{3}\right)=\sup \left\{x_{1}, x_{3}\right\}, \quad \varphi_{2}\left(x_{1}, x_{2}, x_{3}\right)=x_{2},
$$

est une section non dégénérée du préfaisceau $\square_{1} \times \square_{1}$ au-dessus de $\square_{3}$. Néanmoins, le morphisme $\varphi$ n'est pas un monomorphisme (par exemple il égalise la double flèche $\left.((1,1,0),(1,1,1)): \square_{0} \longrightarrow \square_{3}\right)$. Ceci explique que les raisonnements précédents ne s'appliquent pas à ce cas.

\section{Références}

[1] F.A. Al-Agl, R. Brown and R. Steiner, Multiple categories : the equivalence of a globular and a cubical approach, Adv. Math. 170 (2002), no. 1, 71-118. 
[2] R. Antolini, Geometric realisations of cubical sets with connections, and classifying spaces of categories, Appl. Categ. Structures 10 (2002), no. 5, 481-494.

[3] D. Ara, Sur les $\infty$-groupoïdes de Grothendieck, Thèse en préparation sous la direction de G. Maltsiniotis.

[4] R. Brown and P.J. Higgins, On the algebra of cubes, J. Pure Appl. Algebra 21 (1981), no. 3, 233-260.

[5] R. Brown and P.J. Higgins, Colimit theorems for relative homotopy groups, J. Pure Appl. Algebra 22 (1981), no. 1, 11-41.

[6] R. Brown and P.J. Higgins, Tensor products and homotopies for $\omega$-groupoids and crossed complexes, J. Pure Appl. Algebra 47 (1987), no. 1, 1-33.

[7] R. Brown and P.J. Higgins, Cubical abelian groups with connections are equivalent to chain complexes, Homology, Homotopy and Applications 5 (2003), no. 1, $49-52$.

[8] D.-C. Cisinski, Les préfaisceaux comme modèles des types d'homotopie, Astérisque 308 (2006), 1-392.

[9] D.-C. Cisinski and G. Maltsiniotis, La catégorie $\boldsymbol{\Theta}$ de Joyal est une catégorie test, Prépublication (2009).

[10] M. Grandis, Cubical monads and their symmetries, in Proceedings of the Eleventh International Conference of Topology (Trieste, 1993), Rend. Istit. Mat. Univ. Trieste 25 (1993), no. 1-2, 223-262.

[11] M. Grandis and L. Mauri, Cubical sets and their site, Theory Appl. Categories 11 (2003), no. 8, 185-211.

[12] A. Grothendieck, Pursuing stacks, Manuscrit, 1983, à paraître dans Documents Mathématiques.

[13] P.J. Higgins, Thin elements and commutative shells in cubical $\omega$-categories, Theory Applic. Categories 14 (2005), no. 4, 60-74.

[14] P.S. Hirschhorn, Model categories and their localizations, Math. Surveys and Monographs 99, Amer. Math. Soc., Providence, RI, 2003.

[15] M. Hovey, Model categories, Math. Surveys and Monographs 63, Amer. Math. Soc., Providence, RI, 1999.

[16] L. Illusie, Complexe cotangent et déformations I, II, Lecture Notes in Math. 239 and 283, Springer-Verlag, New York, 1971-1972.

[17] S.B. Isaacson, Cubical homotopy theory and monoidal model categories, Thèse sous la direction de M.J. Hopkins (2009).

[18] S.B. Isaacson, Symmetric cubical sets, Prépublication (2009).

[19] A. Joyal, Disks, duality and $\Theta$-categories, Prépublication (1997).

[20] K.H. Kamps and T. Porter, Abstract homotopy and simple homotopy theory, World Sci. Publ., Co., River Edge, NJ, 1997.

[21] D.M. Kan, Abstract homotopy. I, Proc. Nat. Acad. Sci. U.S.A. 41 (1955), 1092-1096. 
[22] G. Maltsiniotis, La théorie de l'homotopie de Grothendieck, Astérisque 301 (2005), 1-140.

[23] D. Quillen, Higher algebraic K-theory. I, in Algebraic K-theory, I : Higher K-theories (Proc. Conf., Battelle Memorial Inst., Seattle, Wash., 1972), Lecture Notes in Math. 341, 85-147, Springer-Verlag, New York, 1973,

[24] A.P. Tonks, Cubical groups which are Kan, J. Pure Appl. Algebra 81 (1992), no. $1,83-87$.

Georges Maltsiniotis maltsin@math.jussieu.fr

Institut de Mathématiques de Jussieu, Université Paris 7 Denis Diderot, Case Postale 7012, 2 place Jussieu, F-75251 Paris Cedex 05, France 